\section{Wayne, Horwich, And Evidential Diversity}

\section{Branden Fitelson ${ }^{\dagger}$}

August 29, 1997

Wayne (1995) critiques the Bayesian explication of the confirmational significance of evidential diversity (CSED) offered by Horwich (1982). Presently, I argue that Wayne's reconstruction of Horwich's account of CSED is uncharitable. As a result, Wayne's criticisms ultimately present no real problem for Horwich. I try to provide a more faithful and charitable rendition of Horwich's account of CSED. Unfortunately, even when Horwich's approach is charitably reconstructed, it is still not completely satisfying.

This paper is an updated and expanded version of Fitelson (1996).

${ }^{\dagger}$ Thanks to Ellery Eells, Malcolm Forster, Leslie Graves, Geoffrey Hellman, Mike Kruse, Patrick Maher, and an anonymous Philosophy of Science referee for their helpful comments and suggestions on earlier versions of this paper.

\section{Introduction}

Wayne (1995) gives one reconstruction of Horwich's (1982) Bayesian account of the value of evidential diversity. He then shows that there are counterexamples to this reconstruction of Horwich's explication of CSED. Such counterexamples would undermine Horwich's account of CSED, if Wayne's reconstruction were a charitable one. Presently, I argue that Wayne's reconstruction of Horwich's account of CSED is uncharitable. As a result, his criticisms are not genuine problems for Horwich.

This does not mean that Horwich's explication of CSED - charitably reconstructed - is unproblematic. On the contrary, after my analysis of Wayne's critique, I discuss several remaining problems for Horwich's account. In the end, I conclude that Horwich's Bayesian explication of CSED is inadequate.

In the final section of the paper, I briefly discuss two recent alternative Bayesian explications of CSED, including a refreshing new account developed in Fitelson (1997a) which is based on a Bayesian account of independent inductive support and its relationship to the diversity and confirmational power of collections of evidence.

\section{Wayne's reconstruction of Horwich's account}

In a typical confirmation theoretic context $\mathcal{C}$, we have a hypothesis under test $H_{1}$ and $n-1$ competing hypotheses $H_{2}, \ldots, H_{n}$, where the $n$ hypotheses are assumed to be mutually exclusive and exhaustive. Wayne's (1995) reconstruction of Horwich's (1982) explication of CSED involves the following three propositions concerning such contexts:

$\left(\mathcal{H}_{1}\right)$ One collection of evidence $E_{1}$ is more confirmationally diverse (c-diverse) than another collection of evidence $E_{2}$ in context $\mathcal{C}$ iff $(\forall i \neq 1)\left[\operatorname{Pr}\left(E_{1} \mid H_{i} \& K_{\mathcal{C}}\right)<\operatorname{Pr}\left(E_{2} \mid H_{i} \& K_{\mathcal{C}}\right)\right]^{1}$

The intuition behind $\mathcal{H}_{1}$ is that the more $c$-diverse collection of evidence is supposed to "rule-out most plausible alternatives" to the hypothesis under test. It is for this reason that Horwich's account has been called 'eliminativist'.

$\left(\mathcal{H}_{2}\right) E_{1}$ confirms $H$ more strongly than $E_{2}$ confirms $H$ if and only if $r\left(H, E_{1}\right)>r\left(H, E_{2}\right)$, where the ratio measure of degree of confirmation $r(H, E)$ is defined as follows: $r(H, E)=_{d f} \frac{\operatorname{Pr}(H \mid E)}{\operatorname{Pr}(H)}$.

${ }^{1}$ Where, the proposition $K_{\mathcal{C}}$ encodes the background knowledge in confirmational context $\mathcal{C}$. Hereafter, I will, for simplicity's sake, drop explicit reference to $K_{\mathcal{C}}$ in probability statements. It is to be understood, of course, that we are uniformly conditioning $\operatorname{Pr}$ on $K_{\mathcal{C}}$, whenever we make a Bayesian confirmational comparison.

${ }^{2}$ Notice that my $\mathcal{H}_{1}$ is sufficient but not necessary for $E_{1}$ 's "ruling-out" most alternatives to $H_{1}$ in $\mathcal{C}$. As we'll see below, this added strength is needed to shore-up Horwich's formal account of CSED. See the APPENDIX for all technical details. 
$\left(\mathcal{H}_{3}\right)$ For every confirmational context $\mathcal{C}$, if $E_{1}$ is more $c$-diverse than $E_{2}$ in $\mathcal{C}$, then $E_{1}$ confirms $H_{1}$ (i.e., the hypothesis under test in $\mathcal{C}$ ) more strongly than $E_{2}$ confirms $H_{1}$ in $\mathcal{C}$.

According to Wayne (1995), $\mathcal{H}_{3}$ captures the kernel of Horwich's account of CSED. In the next section, we will look at a counterexample to $\mathcal{H}_{3}$ due to Wayne (1995).

\section{$3 \quad$ Wayne's counterexample to $\mathcal{H}_{3}$}

Wayne (1995, page 119) asks us to:

... consider a simple context $\mathcal{C}_{w}$ in which only three hypotheses have substantial prior probabilities, $\operatorname{Pr}\left(H_{1}\right)=0.2, \operatorname{Pr}\left(H_{2}\right)=0.2, \operatorname{Pr}\left(H_{3}\right)=0.6$, and two data sets $E_{1}$ and $E_{2}$ such that:

$$
\begin{array}{ll}
\operatorname{Pr}\left(E_{1} \mid H_{1}\right)=0.2 & \operatorname{Pr}\left(E_{2} \mid H_{1}\right)=0.6 \\
\operatorname{Pr}\left(E_{1} \mid H_{2}\right)=0.4 & \operatorname{Pr}\left(E_{2} \mid H_{2}\right)=0.5 \\
\operatorname{Pr}\left(E_{1} \mid H_{3}\right)=0.4 & \operatorname{Pr}\left(E_{2} \mid H_{3}\right)=0.6
\end{array}
$$

This is plainly a paradigm case of $\mathcal{H}_{1}$ : for all $H_{i}, \operatorname{Pr}\left(E_{1} \mid H_{i}\right)$ is significantly less than $\operatorname{Pr}\left(E_{2} \mid H_{i}\right)$. Yet, a straightforward substitution shows that $\mathcal{H}_{3}$ is violated! Thus, we obtain the counterintuitive result that the similar evidence lends a greater boost to the hypothesis under test than does the diverse evidence ... Horwich's account fails to reproduce our most basic intuition about diverse evidence. ${ }^{3}$

Wayne is right about $\mathcal{C}_{w}$ in the following two respects. ${ }^{4}$

(1) In $\mathcal{C}_{w}, E_{1}$ is more $c$-diverse than $E_{2}$.

(2) In $\mathcal{C}_{w}, E_{2}$ confirms $H_{1}$ more strongly than $E_{1}$ confirms $H_{1}$, according to the ratio measure $r$.

Hence, $\mathcal{C}_{w}$ is a legitimate counterexample to $\mathcal{H}_{3}$. In the next section, I will discuss some aspects of Wayne's example that he neglects to mention. Then, I will reflect on what the existence of this counterexample implies - and doesn't imply — about Horwich's account of CSED.

\section{Why Wayne's counterexample is not salient}

\subsection{What Wayne doesn't say about his counterexample}

Here is a fact about Wayne's counterexample to $\mathcal{H}_{3}$ that he neglects to mention.

(3) In $\mathcal{C}_{w}, E_{2}$ confirms $H_{1}$; whereas, $E_{1}$ disconfirms $H_{1}$.

${ }^{3}$ I have taken the liberty of translating this passage from Wayne (1995) into my notation. ${ }^{4}$ See the Appendix for all proofs and counterexamples.
Wayne has certainly described a confirmational context $\mathcal{C}_{w}$ in which a less $c$-diverse data set confirms the hypothesis under test more strongly than a more $c$-diverse data set does. But, as it turns out, $\mathcal{C}_{w}$ is also a context in which the more $c$-diverse evidence disconfirms the hypothesis under test; whereas, the less $c$-diverse evidence confirms the hypothesis under test. What does this mean?

\subsection{Charitably reconstructing Horwich's account}

As far as I can tell, $(3)$ shows that $\mathcal{H}_{3}$ must not be what Horwich has in mind in his explication of CSED. Surely, Horwich would not want to say that more $c$-diverse disconfirmatory evidence should confirm more strongly than less $c$ diverse confirmatory evidence. To say the least, this would not be in the spirit of the Bayesian definition of confirmation.

A more charitable reconstruction of Horwich's account of CSED should add a suitable probabilistic ceteris paribus clause to $\mathcal{H}_{3}$. In such a reconstruction, Wayne's $\mathcal{H}_{3}$ might be replaced by:

$\left(\mathcal{H}_{3}^{\prime}\right)$ If $C P$ then $\mathcal{H}_{3}$.

Where $C P$ is an appropriate probabilistic ceteris paribus clause. Wayne's counterexample teaches us that, at the very least, $C P$ should entail:

$\left(C P_{1}\right)$ Both $E_{1}$ and $E_{2}$ confirm $H_{1}$ in $\mathcal{C}$.

Indeed, $C P_{1}$ would avoid the counterexample raised by Wayne. Moreover, it would insure that $\mathcal{H}_{3}^{\prime}$ does not contradict the Bayesian definition of confirmation (as Wayne's $\mathcal{H}_{3}$ does).

Interestingly, $C P_{1}$ is not a sufficient ceteris paribus clause. For, $C P_{1}$ does not entail $\mathcal{H}_{3} \cdot{ }^{5}$ We will need to make $C P$ substantially stronger than $C P_{1}$ in order to make $\mathcal{H}_{3}^{\prime}$ a theorem of the mathematical theory of probability. There are many ways to define sufficient ceteris paribus clauses in this sense. ${ }^{6}$ Here is one such proposal that I think remains faithful to what Horwich has in mind:

$(C P *) C P_{1}$, and $\operatorname{Pr}\left(E_{1} \mid H_{1}\right)=\operatorname{Pr}\left(E_{2} \mid H_{1}\right)$ in $\mathcal{C}$.

$C P *$ says that $E_{1}$ and $E_{2}$ both confirm $H_{1}$ in $\mathcal{C}$, and that $E_{1}$ and $E_{2}$ are ' $\mathcal{C}$-commensurate', in the sense that the hypothesis under test has the same likelihood (i.e., goodness of fit) with respect to both $E_{1}$ and $E_{2}$ in $\mathcal{C}$. This ceteris

${ }^{5}$ For a relevant counterexample, see the Appendix.

${ }^{6}$ Hellman (1997) proposes the following alternative sufficient ceteris paribus clause:

$(C P \dagger) C P_{1}$, and $\operatorname{Pr}\left(E_{1} \mid H_{1}\right)-\operatorname{Pr}\left(E_{1}\right)=\operatorname{Pr}\left(E_{2} \mid H_{1}\right)-\operatorname{Pr}\left(E_{2}\right)$ in $\mathcal{C}$.

It is true that $C P \dagger$ is sufficient for $\mathcal{H}_{3}$. However, $C P \dagger$ is clearly not the kind of Bayesian proposal that Horwich (1982) has in mind. In Horwich's canonical examples, it is typically assumed that $\operatorname{Pr}\left(E_{1} \mid H_{1}\right)=\operatorname{Pr}\left(E_{2} \mid H_{1}\right)$ (see below for more on this point). Moreover, Horwich wants sets of evidence with greater $c$-diversity to have lesser prior probability (e.g., Horwich wants $\operatorname{Pr}\left(E_{1}\right)<\operatorname{Pr}\left(E_{2}\right)$ in his canonical example). These two constraints jointly entail that $C P \dagger$ does not hold. So, while Hellman's alternative makes sense from a generic Bayesian point of view, it is not a faithful reconstruction of Horwich's Bayesian explication of CSED. 
paribus clause seems to be implicit in Horwich's depiction of the kinds of confirmational contexts he has in mind. Figure 1 shows the kind of confirmational contexts and comparisons that Horwich (1982, pages 119-120) uses as canonical illustrations of his account of CSED.

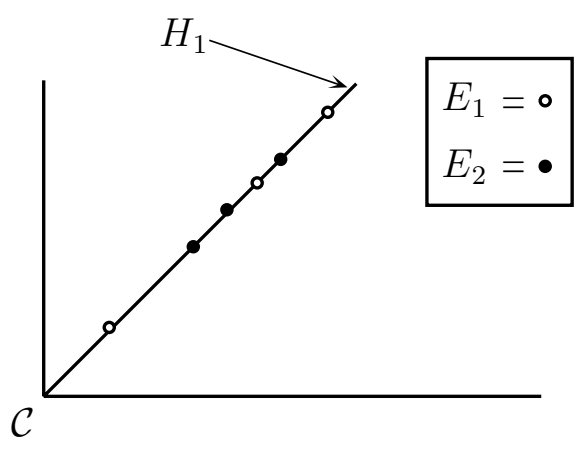

Figure 1: A Canonical Horwichian Example of CSED

Figure 1 depicts a canonical confirmation theoretic context $\mathcal{C}$ in which the hypothesis under test $H_{1}$ fits two data sets $E_{1}$ and $E_{2}$ equally well, in accordance with $C P *$. Moreover, $E_{1}$ is more intuitively diverse ( $i$-diverse) than $E_{2}$, since the abscissa values of $E_{1}$ are more spread-out than the abscissa values of $E_{2}{ }^{7}$ Horwich (1982) seems only to be claiming that — other things being equal (those other things being the likelihoods $\operatorname{Pr}\left(E_{1} \mid H_{1}\right)$ and $\operatorname{Pr}\left(E_{2} \mid H_{1}\right)$ ) - more diverse $^{8}$ sets of evidence (e.g., $\left.E_{1}\right)$ will confirm the hypothesis under test (e.g., $\left.H_{1}\right)$ more strongly than less diverse sets of evidence $\left(e . g ., E_{2}\right)$ will. This is an appropriate time to state the following theorem:

\section{$\left(\mathcal{H}_{3} *\right)$ If $C P *$, then $\mathcal{H}_{3}$.}

Since $\mathcal{H}_{3} *$ is a theorem of the mathematical theory of probability, this reconstruction of Horwich's account is guaranteed to be immune to any formal counterexamples. In this sense, our present reconstruction of Horwich's account is a charitable one. However, even this charitable reconstruction of Horwich's account of CSED has its problems. In the next section, I will briefly discuss some of my remaining worries about Horwich's account of CSED.

${ }^{7}$ This notion of the 'intuitive diversity' ( $i$-diversity) of a data set is never precisely defined by Horwich (1982). But, in canonical curve-fitting contexts, the 'intuitive diversity' of a data set should boil down to some measure of the spread (or variance) of its abscissa values.

${ }^{8} \mathrm{I}$ am being intentionally vague here about which kind of diversity Horwich has in mind. I think Horwich has $i$-diversity in mind; but, he clearly wants this relationship to obtain also with respect to $c$-diversity. I'll try to resolve this important tension below.

\section{Remaining worries about Horwich's account}

\subsection{The relationship between $i$-diversity and $c$-diversity}

Horwich's $\mathcal{H}_{1}$ says that a more $c$-diverse set of evidence $E_{1}$ will tend to "ruleout more of the plausible alternative hypotheses $H_{j \neq 1}$ " than a less $c$-diverse set of evidence $E_{2}$ will. But, when Horwich gives his canonical curve-fitting examples, he appeals to an intuitive sense of diversity ( $i$-diversity) which does not obviously correspond to the formal, confirmational diversity specified in $\mathcal{H}_{1}$. At this point, as natural question to ask is: "What is the relationship between $i$-diversity and $c$-diversity, anyway?"

Ideally, we would like the following general correspondence to obtain between the $i$-diversity and $c$-diversity of data sets:

$\left(\mathcal{H}_{4}\right)$ If $E_{1}$ is more $i$-diverse than $E_{2}$ in $\mathcal{C}$, then $E_{1}$ is more $c$-diverse than $E_{2}$ in $\mathcal{C}$.

If $\mathcal{H}_{4}$ were generally true (i.e., true for all $\mathcal{C}$ ), then all of the intuitive examples of CSED would automatically translate into formal examples of CSED with just the right mathematical properties. And, Horwich's formal account of CSED (i.e., $\mathcal{H}_{1}-\mathcal{H}_{3}^{\prime}$ ) would be vindicated by its ability to match our intuitions about CSED in all cases. Unfortunately, things don't work out quite this nicely.

It turns out that $\mathcal{H}_{4}$ is not generally true. To see this, let's reconsider Horwich's canonical example of CSED, depicted in Figure 1. In this example, $E_{1}$ is more $i$-diverse and more $c$-diverse than $E_{2}$ in $\mathcal{C}$. It is obvious why $E_{1}$ is more $i$-diverse than $E_{2}$ in $\mathcal{C}$ (just inspect the spread of the abcissa values of $E_{1}$ vs $\left.E_{2}\right)$. However, it is not so obvious why $E_{1}$ is more $c$-diverse than $E_{2}$ in $\mathcal{C}$. Horwich claims that $E_{1}$ tends to rule-out more of the plausible alternatives to $H_{1}$ than $E_{2}$ does. I think it is more perspicuous to say instead that $E_{1}$ tends to rule-out more of the simple alternatives to $H_{1}$ than $E_{2}$ does. ${ }^{9}$ Horwich doesn't say exactly how we should measure the 'relative simplicity' of competing hypotheses. We can make some sense out of Horwich's canonical example, if we make the following plausible and common assumption about how to measure the simplicity of a polynomial hypothesis in a curve-fitting context:

$\left(\mathcal{H}_{5}\right)$ The simplicity of a polynomial hypothesis $H$ is equal to the dimensionality of the smallest (non-trivial) family of polynomial

${ }^{9}$ Horwich (1982, pages 121-122) and Horwich (1993, pages 66-67) explains that his account of CSED depends on a substantive Bayesian understanding of the simplicity of statistical hypotheses. Given our reconstruction of Horwich's account of CSED, we can see vividly why this is so. Horwich seems to be assuming that simple hypotheses have some kind of a priori probabilistic advantage over complex hypotheses. This kind of assumption is known as a simplicity postulate. Simplicity postulates are a well-known source of controversy in Bayesian philosophy of science. I won't dwell here on the problematical nature of simplicity postulates, since I think they are a problem for a rather large class of Bayesian accounts of CSED. For an interesting discussion of simplicity postulates in Bayesian confirmation theory, see Popper (1992, Appendix *viii). See, especially, Forster (1995) for a detailed critique of the simplicity postulate in the context of curve-fitting. 
functions of which $H$ is a member. ${ }^{10}$

If we characterize simplicity in this way, we can explain why $E_{1}$ tends to rule-out more of the simple alternatives to $H_{1}$ than $E_{2}$ does in the canonical example depicted in Figure 1. Figure 2 gives us way to picture what's going on in Horwich's canonical example in a rather illuminating and explanatory way. ${ }^{11}$

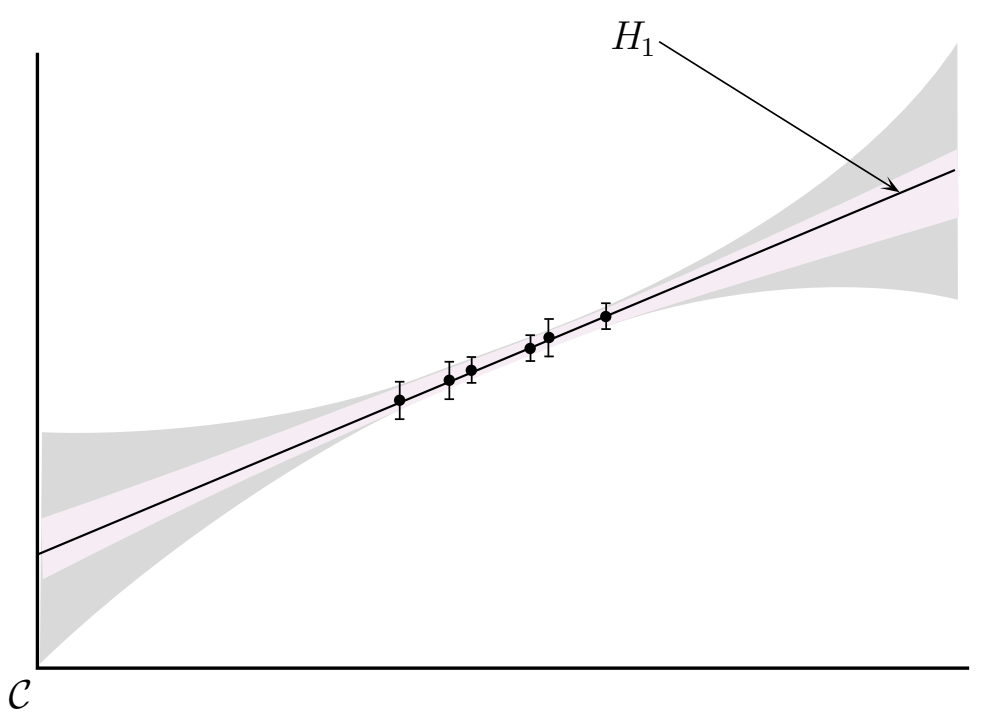

Figure 2: Why Horwich's canonical example has the right formal properties

The lightly shaded area in Figure 2 corresponds to the set of linear hypotheses that are consistent with the data; and, the darkly shaded region corresponds to the set of parabolic hypotheses that are consistent with the data. Now, if we were to 'spread-out' the abscissa values of the data set in Figure 2 - while keeping the likelihood with respect to $H_{1}$ constant, in accordance with $C P *-$ the resulting, more intuitively diverse, data set would end up ruling-out more of the plausible (i.e., simpler) alternative hypotheses than the original data set does. This is because the shaded region (whose area is roughly proportional to the number of simple alternatives to that are consistent with the data) will shrink as we spread out the data set along the linear $H_{1}$. So, in such an example, it is plausible to expect that the more intuitively diverse data set will also be

${ }^{10}$ This is a standard way of measuring the simplicity of hypotheses in curve-fitting contexts Take, for instance, the curve $H: y=x^{2}+2 x$. The smallest (non-trivial) family of polynomials containing $H$ is family PAR: $y=a x^{2}+b x+c$ (where $a, b$, and $c$ are adjustable parameters) PAR). Hence, the 'simplicity value' of $H$ is 3 . As a rule, then, lower dimensionality families contain simpler curves.

${ }^{11}$ Thanks to Malcolm Forster for generating this informative graphic and allowing me to use it for this purpose. more confirmationally diverse in the formal sense of $\mathcal{H}_{1}$. However, this will not generally be the case. In general, whether or not $\mathcal{H}_{4}$ holds will depend on how complex the hypothesis under test is. We can imagine situations in which the hypothesis under test is sufficiently complex relative to its competitors in $\mathcal{C}$. In such situations, increasing the spread (or $i$-diversity) of a data set (in accordance with $C P *$ ) may not automatically increase its confirmational diversity. ${ }^{12}$

To see this, consider a confirmational context $\mathcal{C}^{\prime}$ in which the hypothesis under test $H_{1}$ is a highly complex curve, and has only one competitor in $\mathcal{C}^{\prime}$ : a linear hypothesis $H_{2}$. Now, assume that some data $E_{2}$ set falls exactly on $H_{1}$ in such a way that is inconsistent with $H_{2}$. If we spread out $E_{2}$ in just the right way - in accordance with $C P *$ - to form a more intuitively diverse data set $E_{1}$, we may end up with a data set that is not more confirmationally diverse than $E_{2}$. In fact, depending on how complex $H_{1}$ is (and how cleverly we choose to spread out $E_{2}$ along $\left.H_{1}\right), E_{1}$ may turn-out to be less $c$-diverse than $E_{2}$. For instance, $E_{1}$ might just happen to fall exactly on the linear alternative $H_{2}$. This kind of 'non-canonical' confirmational context - in which a more $i$-diverse data set turns out to be less c-diverse - is pictured below in Figure 3.

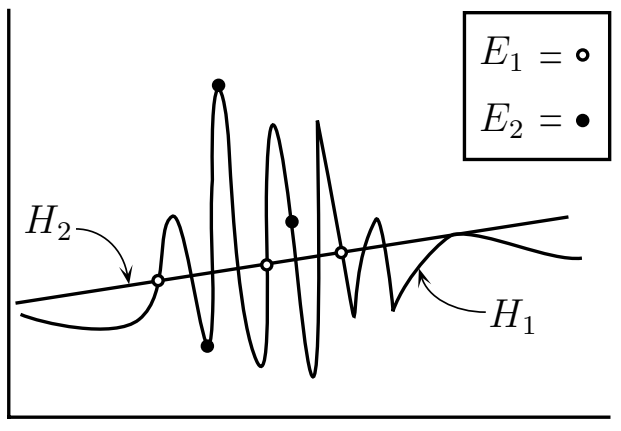

$\mathcal{C}^{\prime}$

Figure 3: Why the truth of $\mathcal{H}_{4}$ depends on the complexity of $H_{1}$

To sum up: Horwich's formal sense of confirmational diversity only corresponds to his intuitive sense of diversity in contexts where the hypothesis under test is a relatively simple hypothesis. If the hypothesis under test is sufficiently complex relative to its competitors, then the connection between Horwich's formal definition of diversity (in $\mathcal{H}_{1}$ ) and the intuitive notion of diversity seen in Horwich's canonical curve-fitting contexts breaks down. Because this connection is essential to the general success of Horwich's approach to explicate our pre-theoretic intuitions about CSED, Horwich's account would seem - at best - to provide an incomplete explication of CSED.

${ }^{12}$ Thanks to Patrick Maher for getting me to see this point clearly. 


\subsection{Horwich's choice of measure of confirmation}

Horwich presupposes that the quotient measure $r$ is an adequate Bayesian measure of degree of confirmation. Several recent authors have argued that $r$ is an inadequate Bayesian measure of confirmation. ${ }^{13}$ While I find some of these reasons for rejecting $r$ rather convincing, I have 'independent' reasons for thinking that $r$ is inadequate. One problem with $r$ is that it is not appropriately sensitive to the distinction between independent and dependent inductive support for a hypothesis. In Fitelson (1997b), I investigate how various measures of confirmation handle (or - in the case of $r$ - fail to handle) the distinction between independent and dependent inductive support. There, I argue that $r$ is not an adequate measure of degree of confirmation, since it fails to properly distinguish between dependent and independent pieces of evidence for a hypothesis. ${ }^{14}$

Luckily for Horwich, however, our reconstruction of his account of CSED is not sensitive to this unfortunate choice of measure of confirmation. It turns out that $\mathcal{H}_{3} *$ remains true for a wide variety of non-equivalent relevance measures of confirmation (see the ApPENDix). ${ }^{15}$

\section{Alternative accounts and future directions}

Carnap (1962) insists that any good Bayesian confirmation theory must be able to adequately explicate CSED. Since then, several Bayesian philosophers of science have answered the call. The two most well-known and popular Bayesian explications of CSED that have appeared in recent years seem to be Horwich's 'eliminativist' explication, and the so-called 'correlation' or 'similarity' explication outlined by Howson and Urbach (1993) and Earman (1992). The 'correlation' approach tries to cache-out the intuition that 'diverse' evidence tends not to be highly self-correlated. I find the 'correlation' account more intuitively appealing than Horwich's, because it attempts to unpack 'diversity' in terms of some kind of independence of the evidence. I carefully examine the 'correlation' explication of CSED in Fitelson (1997a). There, I argue that evidential diversity cannot be properly understood in terms of probabilistic independence of the $e v$ idence simpliciter, as 'correlation' theorists would have us believe. Rather, the increased boost in confirmation provided by more 'diverse' collections of evidence is really a consequence of the elements of such collections of evidence providing independent inductive support for the hypothesis under test.

In Fitelson (1997a), I develop a Bayesian account of independent inductive support, and I use it to construct a refreshing new Bayesian explication of

${ }^{13}$ See, for instance, Gillies (1986), Good (1984), Rosenkrantz (1981), and Schum (1994).
${ }^{14}$ I also argue in Fitelson (1997b) that the difference measure is inadequate. In fact, I argue
in Fitelson (1997b) that only a small class of likelihood-based measures can adequately cope
with independent inductive support. This is bad news for many recent philosophers of science
whose resolutions of various problems in confirmation theory have presupposed inadequate
measures of confirmation.
${ }^{15}$ In this sense, Horwich's account is more robust than the so-called 'correlation' account of
Howson and Urbach (1993). In Fitelson (1997a), I discuss the measure sensitivity of Howson
and Urbach's 'correlation' explication of CSED.

CSED. Unlike Horwich's 'eliminativist' account and/or Howson and Urbach's 'correlation' account, my explication of CSED requires no additional probabilistic 'ceteris paribus clauses'. Furthermore, my account — unlike Howson and Urbach's - holds true for all Bayesian relevance measures of confirmation. 


\section{ApPendix: Proofs and Counterexamples}

\section{A Proofs of (1), (2), and (3)}

\section{A.1 Proof of (1)}

The task at hand is to prove:

(1) In $\mathcal{C}_{w}, E_{1}$ is more $c$-diverse than $E_{2}$.

Proof. Recall that, in Wayne's counterexample context $\mathcal{C}_{w}$, the hypothesis under test $H_{1}$ has only two competitors with non-negligible priors: $H_{2}$ and $H_{3}$. Moreover, Wayne stipulates that, in $\mathcal{C}_{w}$, both:

$$
\begin{aligned}
\operatorname{Pr}\left(E_{1} \mid H_{2}\right) & =0.4 \\
& <\operatorname{Pr}\left(E_{2} \mid H_{2}\right)=0.5, \text { and } \\
\operatorname{Pr}\left(E_{1} \mid H_{3}\right) & =0.4 \\
& <\operatorname{Pr}\left(E_{2} \mid H_{3}\right)=0.6 .
\end{aligned}
$$

In conjunction with the characterization of $c$-diversity given in $\mathcal{H}_{1}$, these two facts about $\mathcal{C}_{w}$ yield the desired result.

\section{A.2 Proof of (2)}

We need to demonstrate that:

(2) In $\mathcal{C}_{w}, E_{2}$ confirms $H_{1}$ more strongly than $E_{1}$ confirms $H_{1}$, according to the ratio measure $r$ (i.e., $r\left(H_{1}, E_{2}\right)>r\left(H_{1}, E_{1}\right)$ ).

Proof. Wayne's description of $\mathcal{C}_{w}$, together with Bayes's Theorem, and the definition of $r$ reported in $\mathcal{H}_{2}$ yields:

$$
\begin{aligned}
r\left(H_{1}, E_{1}\right) & =\frac{\operatorname{Pr}\left(E_{1} \mid H_{1}\right)}{\sum_{i} \operatorname{Pr}\left(E_{1} \mid H_{i}\right) \cdot \operatorname{Pr}\left(H_{i}\right)} \\
& =\frac{0.2}{(0.2 \cdot 0.2)+(0.4 \cdot 0.2)+(0.4 \cdot 0.6)} \\
& \approx 0.555, \text { and } \\
r\left(H_{1}, E_{2}\right) & =\frac{\operatorname{Pr}\left(E_{2} \mid H_{1}\right)}{\sum_{i} \operatorname{Pr}\left(E_{2} \mid H_{i}\right) \cdot \operatorname{Pr}\left(H_{i}\right)} \\
& =\frac{0.6}{(0.6 \cdot 0.2)+(0.5 \cdot 0.2)+(0.6 \cdot 0.6)} \\
& \approx 1.034 .
\end{aligned}
$$

Hence, we have $r\left(H_{1}, E_{2}\right)>r\left(H_{1}, E_{1}\right)$ in $\mathcal{C}_{w}$, as desired.

\section{A.3 Proof of (3)}

Next, we will prove:

(3) In $\mathcal{C}_{w}, E_{2}$ confirms $H_{1}$; whereas, $E_{1}$ disconfirms $H_{1}$.

Proof. According to Bayesian confirmation theory, $E$ confirms $H$ if and only if $r(H, E) \gtrless 1$. This fact about Bayesian confirmation theory, in conjunction with the following two facts about $\mathcal{C}_{w}$ (both of which were proved in the preceding section of this APPENDIX):

$$
r\left(H_{1}, E_{1}\right) \approx 0.555<1, \text { and } r\left(H_{1}, E_{2}\right) \approx 1.034>1,
$$

yields the desired result.

\section{B Proof of Theorem $\mathcal{H}_{3} *$}

In this section, we will not only prove Horwich's $\mathcal{H}_{3} *$, which presupposes the ratio measure $r$ of degree of confirmation; we will also show that $\mathcal{H}_{3} *$ remains true even if we use the difference measure $d$ or the likelihood ratio measure - instead of $r$ - to measure degree of confirmation. Where, the alternative measures of confirmation $d$ and $l$ are defined as follows. ${ }^{16}$

$$
\begin{gathered}
d(H, E)={ }_{d f} \operatorname{Pr}(H \mid E)-\operatorname{Pr}(H) \\
l(H, E)={ }_{d f} \frac{\operatorname{Pr}(E \mid H)}{\operatorname{Pr}(E \mid \bar{H})}
\end{gathered}
$$

This will establish the measure invariance (or measure insensitivity) of our present reconstruction of Horwich's explication of CSED.

\section{B.1 Proof of Horwich's $\mathcal{H}_{3} *$ (the measure $r$ version)}

Below, we prove the following Horwichian version of $\mathcal{H}_{3} *$, which presupposes the measure $r$ of degree of confirmation:

$\left(\mathcal{H}_{3_{r}} *\right)$ If the following probabilistic 'ceteris paribus clause' is satisfied $(C P *) \operatorname{Pr}\left(E_{1} \mid H_{1}\right)=\operatorname{Pr}\left(E_{2} \mid H_{1}\right)$, then:

$$
\begin{gathered}
E_{1} \text { is more } c \text {-diverse than } E_{2} \\
\Downarrow \\
r\left(H_{1}, E_{1}\right)>r\left(H_{1}, E_{2}\right)
\end{gathered}
$$

${ }^{16}$ I use overbars to express negations of propositions (i.e., ' $\bar{X}$ ' stands for ' $n o t-X$ '. 
Proof. The definition of $r$, together with Bayes's Theorem, entails:

$$
r\left(H_{1}, E_{1}\right)=\frac{\operatorname{Pr}\left(E_{1} \mid H_{1}\right)}{\operatorname{Pr}\left(E_{1} \mid H_{1}\right) \cdot \operatorname{Pr}\left(H_{1}\right)+\sum_{i \neq 1} \operatorname{Pr}\left(E_{1} \mid H_{i}\right) \cdot \operatorname{Pr}\left(H_{i}\right)}
$$

Applying Horwich's probabilistic 'ceteris paribus clause' $C P *$, yields the following equation. ${ }^{17}$

$$
r\left(H_{1}, E_{1}\right)=\frac{\operatorname{Pr}\left(E_{2} \mid H_{1}\right)}{\operatorname{Pr}\left(E_{2} \mid H_{1}\right) \cdot \operatorname{Pr}\left(H_{1}\right)+\sum_{i \neq 1} \operatorname{Pr}\left(E_{1} \mid H_{i}\right) \cdot \operatorname{Pr}\left(H_{i}\right)}
$$

Assuming that $E_{1}$ is more $c$-diverse than $E_{2}$ (in the $\mathcal{H}_{1}$ sense), we then have:

$$
r\left(H_{1}, E_{1}\right)>\frac{\operatorname{Pr}\left(E_{2} \mid H_{1}\right)}{\operatorname{Pr}\left(E_{2} \mid H_{1}\right) \cdot \operatorname{Pr}\left(H_{1}\right)+\sum_{i \neq 1} \operatorname{Pr}\left(E_{2} \mid H_{i}\right) \cdot \operatorname{Pr}\left(H_{i}\right)}
$$

Applying the definition of $r$ and Bayes's Theorem gives: $r\left(H_{1}, E_{1}\right)>r\left(H_{1}, E_{2}\right)$, which completes the proof of $\mathcal{H}_{3_{r}} *$.

\section{B.2 Proof of the measure $d$ version of $\mathcal{H}_{3} *$}

Below, we prove the following alternative version of $\mathcal{H}_{3} *$, which presupposes the difference measure $d$ of degree of confirmation:

$\left(\mathcal{H}_{3 d} *\right)$ If the following probabilistic 'ceteris paribus clause' is satisfied $(C P *) \operatorname{Pr}\left(E_{1} \mid H_{1}\right)=\operatorname{Pr}\left(E_{2} \mid H_{1}\right)$, then:

$E_{1}$ is more $c$-diverse than $E_{2}$

$$
\begin{gathered}
\Downarrow \\
d\left(H_{1}, E_{1}\right)>d\left(H_{1}, E_{2}\right)
\end{gathered}
$$

Proof. It turns out that $\mathcal{H}_{3_{d}} *$ is a straightforward corollary of $\mathcal{H}_{3_{r}} *$. Simple algebra shows that $d\left(H_{1}, E_{1}\right)>d\left(H_{1}, E_{2}\right)$ if and only if $r\left(H_{1}, E_{1}\right)>r\left(H_{1}, E_{2}\right)$. Hence, any sufficient condition for $r\left(H_{1}, E_{1}\right)>r\left(H_{1}, E_{2}\right)$ is automatically a sufficient condition for $d\left(H_{1}, E_{1}\right)>d\left(H_{1}, E_{2}\right)$, and vice versa.

${ }^{17}$ Notice that the assumption $\operatorname{Pr}\left(E_{1} \mid H_{1}\right)=\operatorname{Pr}\left(E_{2} \mid H_{1}\right)$ in $\mathcal{C}$ is, by itself, sufficient for $\mathcal{H}_{3} *$ However, while we make no use of the first conjunct of $C P *$ in our proof of $\mathcal{H}_{3} *$, we still must include the assumption that both $E_{1}$ and $E_{2}$ confirm $H_{1}$ in $\mathcal{C}$, in order to avoid spurious counterexamples of the kind constructed by Wayne.

\section{B.3 Proof of the measure $l$ version of $\mathcal{H}_{3} *$}

Below, we prove the following alternative version of $\mathcal{H}_{3} *$, which presupposes the likelihood ratio measure $l$ of degree of confirmation:

$\left(\mathcal{H}_{3_{l}} *\right)$ If the following probabilistic 'ceteris paribus clause' is satisfied $(C P *) \operatorname{Pr}\left(E_{1} \mid H_{1}\right)=\operatorname{Pr}\left(E_{2} \mid H_{1}\right)$, then:

$E_{1}$ is more $c$-diverse than $E_{2}$

$$
l\left(H_{1}, E_{1}\right)>l\left(H_{1}, E_{2}\right)
$$

Proof. By the definition of $l$, we have the following biconditional:

$$
l\left(H_{1}, E_{1}\right)>l\left(H_{1}, E_{2}\right) \Longleftrightarrow \frac{\operatorname{Pr}\left(E_{1} \mid H_{1}\right)}{\operatorname{Pr}\left(E_{1} \mid \bar{H}_{1}\right)}>\frac{\operatorname{Pr}\left(E_{2} \mid H_{1}\right)}{\operatorname{Pr}\left(E_{2} \mid \bar{H}_{1}\right)}
$$

If we assume that the 'ceteris paribus clause' $(C P *)$ is satisfied, this becomes:

$$
l\left(H_{1}, E_{1}\right)>l\left(H_{1}, E_{2}\right) \Longleftrightarrow \frac{1}{\operatorname{Pr}\left(E_{1} \mid \bar{H}_{1}\right)}>\frac{1}{\operatorname{Pr}\left(E_{2} \mid \bar{H}_{1}\right)}
$$

Applying a little algebra, we then have the following result:

$$
(C P *) \Longrightarrow\left[l\left(H_{1}, E_{1}\right)>l\left(H_{1}, E_{2}\right) \Longleftrightarrow \operatorname{Pr}\left(E_{1} \mid \bar{H}_{1}\right)<\operatorname{Pr}\left(E_{2} \mid \bar{H}_{1}\right)\right]
$$

Now, from the nature of confirmational contexts, we know that $\bar{H}_{1}$ is logically equivalent to $\bigvee H_{i \neq 1}$, where the $H_{i \neq 1}$ are mutually exclusive. Hence, from the probability calculus, we may infer both:

$$
\operatorname{Pr}\left(E_{1} \mid \bar{H}_{1}\right)=\frac{\sum_{i \neq 1} \operatorname{Pr}\left(E_{1} \mid H_{i}\right) \cdot \operatorname{Pr}\left(H_{i}\right)}{\sum_{i \neq 1} \operatorname{Pr}\left(H_{i}\right)},
$$

and

$$
\operatorname{Pr}\left(E_{2} \mid \bar{H}_{1}\right)=\frac{\sum_{i \neq 1} \operatorname{Pr}\left(E_{2} \mid H_{i}\right) \cdot \operatorname{Pr}\left(H_{i}\right)}{\sum_{i \neq 1} \operatorname{Pr}\left(H_{i}\right)} .
$$

From which (with some algebraic manipulation), we may obtain:

$$
(\forall i \neq 1)\left[\operatorname{Pr}\left(E_{1} \mid H_{i}\right)<\operatorname{Pr}\left(E_{2} \mid H_{i}\right)\right] \Longrightarrow \operatorname{Pr}\left(E_{1} \mid \bar{H}_{1}\right)<\operatorname{Pr}\left(E_{2} \mid \bar{H}_{1}\right)
$$

But, the antecedent of $(* *)$ just says that $E_{1}$ is more $c$-diverse than $E_{2}$, in the sense of $\mathcal{H}_{1}$. Therefore, $(*)$ and $(* *)$ jointly entail $\mathcal{H}_{3_{l}} *$. 


\section{Counterexample to $C P_{1} \Longrightarrow \mathcal{H}_{3}$}

In this section, we show (by generating a concrete counterexample) that:

$$
C P_{1} \nRightarrow \mathcal{H}_{3} \text {. }
$$

Proof. Consider a simple context ${ }^{18} \mathcal{C}_{w_{1}}$ in which only three hypotheses have substantial prior probabilities, $\operatorname{Pr}\left(H_{1}\right)=0.2, \operatorname{Pr}\left(H_{2}\right)=0.2, \operatorname{Pr}\left(H_{3}\right)=0.6$, and two data sets $E_{1}$ and $E_{2}$ such that:

$$
\begin{array}{ll}
\operatorname{Pr}\left(E_{1} \mid H_{1}\right)=0.41 & \operatorname{Pr}\left(E_{2} \mid H_{1}\right)=0.6 \\
\operatorname{Pr}\left(E_{1} \mid H_{2}\right)=0.4 & \operatorname{Pr}\left(E_{2} \mid H_{2}\right)=0.5 \\
\operatorname{Pr}\left(E_{1} \mid H_{3}\right)=0.4 & \operatorname{Pr}\left(E_{2} \mid H_{3}\right)=0.6
\end{array}
$$

This is plainly a case in which $E_{1}$ is more $c$-diverse than $E_{2}$, in the sense of $\mathcal{H}_{1}$ : for all $H_{i}, \operatorname{Pr}\left(E_{1} \mid H_{i}\right)$ is significantly less than $\operatorname{Pr}\left(E_{2} \mid H_{i}\right)$. Moreover, this is also a case in which the probabilistic 'ceteris paribus clause' $C P_{1}$ holds. As the following calculations show, both $E_{1}$ and $E_{2}$ confirm $H_{1}$ in $\mathcal{C}_{w_{1}}$.

$$
\begin{aligned}
\operatorname{Pr}\left(H_{1} \mid E_{1}\right) & =\frac{\operatorname{Pr}\left(E_{1} \mid H_{1}\right) \cdot \operatorname{Pr}\left(H_{1}\right)}{\sum_{i} \operatorname{Pr}\left(E_{1} \mid H_{i}\right) \cdot \operatorname{Pr}\left(H_{i}\right)} \\
& =\frac{0.41 \cdot 0.2}{(0.41 \cdot 0.2)+(0.4 \cdot 0.2)+(0.4 \cdot 0.6)} \\
& \approx 0.204 \\
& >\operatorname{Pr}\left(H_{1}\right)=0.2, \text { and } \\
\operatorname{Pr}\left(H_{1} \mid E_{2}\right) & =\frac{\operatorname{Pr}\left(E_{2} \mid H_{1}\right) \cdot \operatorname{Pr}\left(H_{1}\right)}{\sum_{i} \operatorname{Pr}\left(E_{2} \mid H_{i}\right) \cdot \operatorname{Pr}\left(H_{i}\right)} \\
& =\frac{0.6 \cdot 0.2}{(0.6 \cdot 0.2)+(0.5 \cdot 0.2)+(0.6 \cdot 0.6)} \\
& \approx 0.207 \\
& >\operatorname{Pr}\left(H_{1}\right)=0.2 .
\end{aligned}
$$

Finally, $\mathcal{C}_{w_{1}}$ is such that $E_{2}$ (the less $c$-diverse collection of evidence) confirms $H_{1}$ more strongly than $E_{1}$ (the more $c$-diverse collection of evidence), according to all three Bayesian relevance measures $r, d$, and $l$. This follows from the fact that $\operatorname{Pr}\left(H_{1} \mid E_{2}\right)>\operatorname{Pr}\left(H_{1} \mid E_{1}\right)$ in $\mathcal{C}_{w_{1}}$ (see above), and the proofs given in the previous section concerning the sufficiency of $\operatorname{Pr}\left(H_{1} \mid E_{2}\right)>\operatorname{Pr}\left(H_{1} \mid E_{1}\right)$ for $\mathfrak{c}\left(H_{1}, E_{2}\right)>\mathfrak{c}\left(H_{1}, E_{1}\right)$, where $\mathfrak{c}$ is any of the three Bayesian relevance measures of confirmation $r, d$, or $l$. Therefore, $\mathcal{C}_{w_{1}}$ is a counterexample to $C P_{1} \Longrightarrow \mathcal{H}_{3}$.

${ }^{18}$ Note: $\mathcal{C}_{w_{1}}$ is just a slight modification of Wayne's $\mathcal{C}_{w}$. I have just changed the value of $\operatorname{Pr}\left(E_{1} \mid H_{1}\right)$ in Wayne's $\mathcal{C}_{w}$ from 0.2 to 0.41 , while leaving the rest of $\mathcal{C}_{w}$ unchanged.

\section{References}

Carnap, R. (1962). The Logical Foundations of Probability, 2nd ed. University of Chicago Press.

Earman, J. (1992). Bayes or Bust: A Critical Examination of Bayesian Confirmation Theory. The MIT Press.

Fitelson, B. (1996). Wayne, Horwich, and Evidential Diversity. Philosophy of Science 63, 652-660.

Fitelson, B. (1997a). A Bayesian Account of Independent Inductive Support with an Application to Evidential Diversity. Unpublished manuscript (completed for Preliminary Examination).

Fitelson, B. (1997b). Independent Inductive Support and Measures of Confirmation. Unpublished manuscript (completed for Preliminary Examination).

Forster, M. (1995). Bayes and Bust: Simplicity as a Problem for a Probabilist's Approach to Confirmation. The British Journal for the Philosophy of Science $46,399-424$

Gillies, D. (1986). In Defense of the Popper-Miller Argument. Philosophy of Science 53, 110-113.

Good, I. (1984). The Best Explicatum for Weight of Evidence. Journal of Statistical Computation and Simulation 19, 294-299.

Hellman, G. (1997). Bayes and Beyond. Forthcoming in Philosophy of Science.

Horwich, P. (1982). Probability and Evidence. Cambridge University Press.

Horwich, P. (1993). Wittgensteinian Bayesianism. In Midwest Studies in Philosophy Volume XVIII: Philosophy of Science. University of Notre Dame Press.

Howson, C. and P. Urbach (1993). Scientific Reasoning: The Bayesian Approach. Open Court.

Popper, K. (1992). The Logic of Scientific Discovery. Routledge.

Rosenkrantz, R. (1981). Foundations and Applications of Inductive Probability. Ridgeview.

Schum, D. (1994). The Evidential Foundations of Probabilistic Reasoning. John Wiley \& Sons.

Wayne, A. (1995). Bayesianism and Diverse Evidence. Philosophy of Science 62, 111-121. 